\title{
Minimal fully adaptive wormhole routing on hypercubes
}

\author{
Chiu-Chuan Lin, Ferng-Ching Lin * \\ Department of Computer Science and Information Engineering, National Taiwan University, Taipei, Taiwan, ROC
}

(Communicated by K. Ikeda; received 11 August 1993; revised 9 March 1994)

\begin{abstract}
Wormhole routing is an advanced switching technique used in new generation multicomputers. Since such a machine may suffer serious performance degradation under heavy or uneven traffic load, an adaptive routing method is particularly called upon. In minimal fully adaptive routing, the paths between any source and destination pair to be used are exactly all the shortest paths. We propose in this paper a minimal fully adaptive routing algorithm for $n$-dimensional hypercube with $|(n+1) / 2|$ virtual channels per physical channel.
\end{abstract}

Key words: Hypercube multicomputer; Wormhole routing; Virtual channel; Fully adaptive; Parallel processing

\section{Introduction}

A distributed-memory multicomputer system achieves interprocessor communication by message passing. Communication latency has significant influence on the performance of such machines, especially when the number of processors scales up. Various interconnection network topologies have been suggested in the literature to address the communication issues like degree, diameter, congestion, symmetry, connectivity and fault tolerance. Another aspect is the switching technique, Store-and-forward switching is used in the first generation parallel machines. In order to decrease the amount of time spent in transmitting data, virtual cut-through switching was introduced [4]. In virtual cut-through, a message is buffered at an indeterminate node only if the

\footnotetext{
* Corresponding author.
}

next channel is busy. In order to avoid buffering messages altogether, wormhole switching $[2,6]$ has been adopted in advanced multiprocessor systems, e.g. Ametek 2010 (Symult), Ncube-2, and iWARP.

With wormhole routing, a message is decomposed into a sequence of flits (flow control digits). As the message moves, its flits spread over several nodes like a "worm". This worm stops moving and remains there if it is blocked by another worm until that worm moves away. It is possible for the first flit of a worm to arrive at the destination node before its last flit leaves the source node. In realizing wormhole switching, a small buffer is associated with each channel to store a flit. The header flit of a message contains all the needed routing information to decide the selection of the next channel on the route. If the buffer of the next channel is free, the header flit moves to that buffer. The subsequent flits advance themselves in a pipelined fashion. 
A routing strategy is oblivious (or deterministic) if the route of a message is completely determined by its source-address and destination-address pair. It is adaptive if alternative paths between the same pair of nodes are allowed to reduce communication latency. Adaptive routing has two advantages: (1) Local congestion can be diffused by exploiting alternative paths to a destination. (2) If any faulty component is encountered along one path, another path can be taken to preserve communication. An adaptive routing method is called minimal if only shortest paths can be used. A minimal adaptive routing is called fully adaptive if each of the shortest paths between any source and destination pair can be used, otherwise it is partially adaptive.

A required condition for wormhole routing is deadlock-free. Deadlock happens when the header flit of a message is destined for a buffer that is occupied by another message whose header flit in turns waits for another message, and this forms a circular wait situation. Deadlock-free wormhole routing algorithms can be designed based on the concept of virtual channels. Using virtual channels, Dally and Seitz [3] first proposed some deadlock-free routing algorithms for unidirectional $k$-ary $n$-cube, cube-connected cycles and shuffle-exchange networks.

The application of virtual channels extends beyond deadlock avoidance, however. They are a versatile mechanism that can be applied to solve a number of network problems. Especially, virtual channels can be used to provide adaptive routing in wormhole switching networks. Yantchev and Jesshope [7] proposed a fully adaptive double-xy routing algorithm for $2 \mathrm{D}$ mesh. Their algorithm requires two virtual channels per physical channel. Dally [1] proposed a double-y routing algorithm that is also fully adaptive. It requires two virtual channels per physical channel along only one of the two dimensions. Linder and Harden [5] extended Dally and Seitz's idea [3] to design adaptive and fault-tolerant routings for three types of $k$-ary $n$-cubes. Their routing algorithms are fully adaptive but the number of virtual channels used in each physical channel is exponential in $n$.

Hypercube has been a popular network topology since it was proposed. There are many nice properties that make hypercube a powerful network. In addition to moderate average distance and diameter, it possesses symmetries, recursive structure and high connectivity to deal with fault tolerance. Many network topologies can be embedded onto hypercube with little effort. Fully adaptive wormhole routing on $n$-dimensional hypercube needs $2^{n-1}$ or $2^{n-2}$ virtual channels per physical channel under Linder and Harden's algorithm [5]. A better routing method by Dally [6] needs $2^{i-1}$ virtual channels for each of the $i$ th dimensional physical channels. In this paper, we propose a novel fully adaptive routing algorithm for hypercube that uses only $[(n+1) / 2]$ virtual channels for each physical channel.

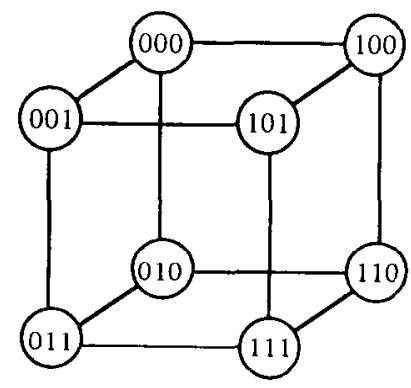

(a) $Q_{3}$

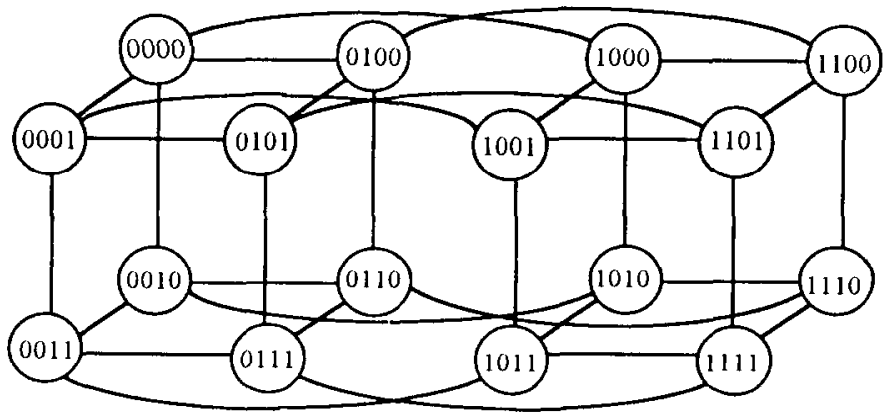

(b) $Q_{4}$

Fig. 1. Hypercube networks. 


\section{Notations}

An $n$-dimensional hypercube $Q_{n}$ has $N=2^{n}$ processors. Each processor is named by an $n$-bit binary string. Two processors are linked by a bidirectional channel if and only if their binary strings differ in precisely one bit. Specifically, processor $a_{n-1} a_{n-2} \ldots a_{i} \ldots a_{0}$ is connected to processor $a_{n-1} a_{n-2} \ldots \bar{a}_{i} \ldots a_{0}$ through a bidirectional channel of dimension $i$. Fig. 1 depicts a 3-dimensional hypercube and a 4-dimensional hypercube.

The exclusive-or of two binary strings $u=$ $u_{n-1} u_{n-2} \ldots u_{0}$ and $v=v_{n-1} v_{n-2} \ldots v_{0}$ is defined by $u \oplus v=r_{n-1} r_{n-2} \ldots r_{0}$, where $r_{i}=0$ if $u_{i}=v_{i}$ and $r_{i}=1$ if $u_{i} \neq v_{i}$, for $0 \leqslant i<n$. We define the dimension set $D(u, v)$ to be the set of dimensions where binary strings $u$ and $v$ differ, i.e. $D(u, v)$ $=\left\{i \mid r_{i}=1,0 \leqslant i<n\right\}$. Let $e^{k}$ denote the binary string whose bits are all 0 except the $k$ th bit. Let $(u, p)$ be a channel of dimension $k$, i.e. $u \oplus p=$ $e^{k}$. We call the direction of $(u, p)$ to be positive if $u_{k}=0$, otherwise it is negative.

Virtual channels are logical channels to share a physical channel. They are time multiplexed over that physical channel. A flit buffer must be maintained in the processor for each virtual channel. We use $(u, p)_{j}$ to denote the $j$ th virtual channel of channel $(u, p)$. The set of all $j$ th virtual channels in the hypercube network is called the $j$ th virtual network.

A well-known sufficient condition for a routing algorithm to be deadlock-free is that the resource dependence graph (RDG) of the network is acyclic. When dealing with wormhole routing using virtual channels, a node in the RDG represents a virtual channel (or equivalently a flit buffer) of a physical channel. There is a directed edge from node $A$ to node $B$ in the RDG if a message can be passed first through the virtual channel represented by $A$ and then through the virtual channel represented by $B$.

\section{Fully adaptive routing on hypercube}

An adaptive routing algorithm can be defined in two parts. The first part is an adaptive set function that determines the set of virtual channels for the header flit to go one step further based on the current processor address, destination processor address and some routing information the header flit possesses. The set of virtual channels determined will be called the adaptive set (AS). The second part is an output selection policy that selects a virtual channel from the adaptive set to route according to the network congestion information. This decomposition is critical, because the adaptive set function may decide whether the routing algorithm is deadlock-free or not, and the output sclcction policy affects the communication performance.

To avoid deadlock and provide fully adaptive routing in an $n$-dimensional hypercube, we split each physical channel into $\lceil(n+1) / 2\rceil$ virtual channels. These virtual channels form $[(n+1) / 2 \mid$ virtual networks. Initially, the message is routed in the 0th virtual network. During the routing, a message may transfer to the next virtual network only when the previous channel direction is negative and the message chooses positive direction to route in the next step. The message always gets closer to the destination in the routing steps.

For example, suppose we need to route a message from 011 to 100 in a 3-dimensional hypercube. There are 3 ! $(=6)$ shortest paths between the source and destination pair. We split each physical channel into $[(3+1) / 2\rceil=2$ virtual channels. The six possible virtual channel sequences provided by the routing algorithm are:

$$
\begin{aligned}
& (011,111)_{0} \rightarrow(111,101)_{0} \rightarrow(101,100)_{0} \\
& (011,111)_{0} \rightarrow(111,110)_{0} \rightarrow(110,100)_{0} \\
& (011,001)_{0} \rightarrow(001,101)_{1}>(101,100)_{1} \\
& (011,001)_{0} \rightarrow(001,000)_{0} \rightarrow(000,100)_{1} \\
& (011,010)_{0} \rightarrow(010,110)_{1} \rightarrow(110,100)_{1} \\
& (011,010)_{0} \rightarrow(010,000)_{0} \rightarrow(000,100)_{1}
\end{aligned}
$$

Since the channel direction of the previous routing step will affect the choice of the virtual network to route in the following routing step, the direction information must be sent along with the header flit. The routing algorithm first computes the AS according to the current address, the destination address, the virtual nctwork and 
the direction of the virtual channel used in the pervious routing step. If the current processor is the source processor, we assume the virtual network to be the 0th and the previous channel direction to be positive. After the AS is determined, the output selection policy randomly selects a free virtual channel from it to route the message. If all virtual channels in the current AS are blocked, the header flit has to wait until one of them is free. The routing algorithm is formally described below.

\section{Algorithm ADAPTIVE_ROUTE;}

$l^{*}$ Node $u$ receives a header flit $(v, m s g, v n$, direction $)^{*} /$

$l^{*}$ If current node $u$ is the source, the header flit is $(v, m s g, 0, \text { pos })^{*} /$

if $u=v$ then stop

$f^{*}$ The destination is reached $* /$

else

begin

$$
\text { if } \text { direction }=\text { pos }
$$

then $\mathrm{AS}=\left\{(u, p)_{v n} \mid u \oplus p=e^{k}, k \in D(u, v)\right\}$ else $\mathrm{AS}=\left\{(u, p)_{v n} \mid u \oplus p=e^{k}, u_{k}=1, k \in\right.$ $D(u, v)\} \cup\left\{(u, p)_{v n+1} \mid u \oplus p=e^{k}, u_{k}=0\right.$, $k \in D(u, v)\}$;

randomly select a free virtual channel $(u, p)_{v n^{\prime}}$ in AS, where $u \oplus p=e^{k}$;

if $u_{k}=0$ then direction $=$ pos else direction $=$ neg;

send header flit ( $v, m s g, v n^{\prime}$, direction) along

end $(u, p)_{v n^{\prime}}$

Theorem 1. The routing algorithm ADAPTIVE ROUTE uses $\lceil(n+1) / 2\rceil$ virtual channels for each physical channel in $Q_{n}$.

Proof. A message always starts to route in the first virtual network. It transfers to the next virtual network only when the virtual channel used in the previous routing step is in negative direction and the next channel selected is in positive direction. The worst case occurs when a message first routes along a negative channel and then alternately routes in positive and negative channels. Since the maximum length of shortest paths in $Q_{n}$ is $n$, the number of virtual networks needed is $\lceil(n+1) / 2\rceil$.

Theorem 2. The routing algorithm ADAPTIVE_ROUTE is deadlock-free.

Proof. It is sufficient to prove that the RDG of the routing algorithm is acyclic. From the description of the algorithm, a message may change virtual networks in ascending order only. Therefore, any directed cycle in the RDG must be composed of virtual channels in the same virtual network.

Suppose there is a directed cycle $C$ formed by virtual channels in the $j$ th virtual network in the RDG. If $(u, p)_{j}$ is a positive channel of dimension $k$ in $C$, then there must be a negative channel $\left(u^{\prime}, p^{\prime}\right)_{j}$ is dimension $k$ in $C$. This implies that $C$ contains a node pair $(x, y)_{j}$ and $(y$, $z)_{j}$ where $(x, y)_{j}$ is adjacent to $(y, z)_{j}$, and $(x, y)_{j}$ has negative direction and $(y, z)_{j}$ has positive direction. This cannot happen due to the nature of the algorithm. Similar argument holds for $(u$, $p)_{i}$ being negative. So we can conclude that the RDG is acyclic and hence ADAPTIVE_ROUTE is deadlock-free.

Theorem 3. The routing algorithm ADAPTIVE_ROUTE is minimal fully adaptive.

Proof. No matter the channel direction used in the previous routing step is positive or negative, the AS determined always contains a virtual channel to route next for each dimension in $D(u$, $v$ ), where $u$ is the current processor and $v$ is the destination processor. So it is easy to see that the routing is minimal fully adaptive.

\section{Concluding remarks}

We have presented a deadlock-free, minimal fully adaptive wormhole routing algorithm for $n$-dimensional hypercube multicomputer using $\lceil(n+1) / 2\rceil$ virtual channcls per physical channcl. 
This number of virtual channels used is a great improvement over the results of Dally [6] and Linder and Harden [5]. We conjecture that this number of virtual channels is optimal for hypercube. It is easy to extend our algorithm to $k$-ary $n$-cube and the number of virtual channels used per physical channel there is $[(\mid k / 2\rfloor n+1) / 2]$. For general multicomputer networks, it is desirable to derive a fully adaptive wormhole routing strategy using $\lceil(d+1) / 2\rceil$ virtual channels for each physical channel, where $d$ is the diameter of the network.

\section{Acknowledgment}

The authors wish to thank one of the referees for his suggestion for improving the presentation of the proof of Theorem 2 .

\section{References}

[1] W.J. Dally, Fine-grain concurrent computers, in: Proc. 3rd Symp. on Hypercube Concurrent Computers and Applications (1988) 2-12.

[2] W.J. Dally and C.L. Seitz, The torus routing chip, Distributed Comput. 1 (3) (1986) 187-196.

[3] W.J. Dally and C.L. Seitz, Deadlock-free message routing in multiprocessor interconnection networks, IEEE Trans. Comput. 36 (5) (1987) 547-553.

[4] P. Kermani and L. Kleinrock, Virtual cut-through: A new computer communication switching technique, Computer Networks 3 (1979) 267-286.

[5] D.H. Linder and J.C. Harden, An adaptive and fault tolerant wormhole routing strategy for $k$-ary $n$-cubes, IEEE Trans. Comput. 40 (1) (1991) 2-12.

[6] R. Suaya and G. Rirtwistle, eds., VT.SI and Parallel Computation (Morgan Kaufmann Publishers, San Mateo, CA, 1990).

[7] J. Yantehev and C.R. Jesshope, Adaptive, low latency, deadlock-free packet routing for networks of processors, IEE Proceedings-E 136 (3) (1989) 178-186. 